\title{
El aprendizaje basado en problemas en la enseñanza de la urología. Modelo de la Facultad de Medicina de la Universidad de Castilla-La Mancha
}

\author{
A.S. Salinas Sánchez*, I. Hernández Millán*, J.A. Virseda Rodríguez *, \\ M. Segura Martín, J.G. Lorenzo Romero, J.M. Giménez Bachs, M ${ }^{a}$.J. Donate Moreno, \\ R. Ruiz Mondéjar, L. Cañamares Pabolaza, L. Polo Ruiz, J.M. Pastor Guzmán, \\ B. Martínez Córcoles, M. Martínez Martín
} Servicio de Urología del Complejo Hospitalario y Universitario de Albacete. *Profesor Asociado de Urología.
Departamento de Ciencias Médicas. Facultad de Medicina. Universidad de Castilla-La Mancha.

Actas Urol Esp 2005; 29 (1): 8-15

\section{RESUMEN}

EL APRENDIZAJE BASADO EN PROBLEMAS EN LA ENSEÑANZA DE LA UROLOGÍA. MODELO DE LA FACULTAD DE MEDICINA DE LA UNIVERSIDAD DE CASTILLA-LA MANCHA

Las nuevas necesidades derivadas de los actuales cambios sociales, económicos, tecnológicos, científicos, etc..., han hecho que distintos organismos hayan sugerido a los estamentos educativos la necesidad de desarrollar cambios en las estrategias educativas orientados al desarrollo de un profesional eficaz con competencias adaptadas a estas necesidades.

Dentro de las estrategias "modernas" se encuentra la enseñanza basada en problemas o aprendizaje por problemas (PBL), mediante la cual el estudiante busca y selecciona la información, razona e integra los conocimientos previos y adquiridos, dando finalmente unas posibilidades diagnósticas y terapéuticas al problema planteado, tal y como se va a enfrentar en su actividad profesional. Los conocimientos previos y la actividad constituyen los pilares fundamentales del aprendizaje. El PBL incorpora algunos de los aspectos de la psicología cognitiva, modelo cuya parte principal determina la naturaleza de las estructuras del conocimiento que se encuentran en la memoria activa, en los procesos de almacenamiento y recuperación de la información y los diversos factores que lo activan.

La urologia en la Facultad de Medicina de la Universidad de Castilla-La Mancha forma parte de una asignatura troncal (Patología Médica y Quirúrgica II) que se imparte durante el quinto curso. La urología se desarrolla integrada con nefrología. El número de alumnos por curso es aproximadamente 75 divididos en 5 grupos. La "rotación" dura seis semanas durante las cuales los estudiantes tienen una media de 2 horas diarias de teoría (ya sean de nefrología y/o urología) y el resto son rotatorias por distintas actividades: tres semanas en nefrología y tres semanas en urología. Al finalizar la rotación los estudiantes realizan un examen teórico conjunto con 100 preguntas de respuestas múltiple (50 de urología) y un examen práctico de las habilidades. A final de curso existe otra prueba práctica (OSCE: examen clínico objetivo y estructurado) con la utilización de pacientes estandarizados, debiendo el profesor evaluar de forma directa el nivel de competencias adquiridas ante un "caso real".

Palabras Clave: Aprendizaje basado en problemas. Enseñanza. Docencia. Pregrado. Urología.

\section{ABSTRACT \\ PROBLEM-BASED LEARNING IN UROLOGY TRAINING. THE FACULTY OF MEDICINE OF THE UNIVERSIDAD DE CASTILLA-LA MANCHA MODEL}

Ongoing changes in the social, economic, technological and scientific realms have generated new needs and led various organizations to suggest that educational institutions should reorient their educational strategies toward developing effective professionals with the skills to meet these needs.

These "modern" strategies include problem-based learning, in which the student seeks and selects information, analyzes the data obtained, integrates both prior and newly acquired knowledge, and, finally, offers diagnostic and therapeutic options to resolve the problem posed, as would occur in professional practice. With this approach, prior skills and practical experience form the foundation of learning. Problem-based learning incorporates some aspects of cognitive psychology, a model that mainly centers on the nature of the knowledge structures found in active memory, the processes involved in information storage and retrieval and the various factors that activate these processes.

At the Faculty of Medicine of the Universidad de Castilla-La Mancha, urology is part of a core subject (Medical and Surgical Pathology II) taught in the fifth year of coursework together with nephrology. Each course includes approximately 75 students, divided into five groups. The rotation lasts six weeks, with students spending a mean of two hours a day on theory (nephrology and/or urology) and the remaining time on rotations in the various activities: three weeks in nephrology and three weeks in urology. Upon completion of the rotation, the students write a combined theoretical examination with 100 multiple-choice questions (50 on urology) and take a practical skills examination. At the end of the course, another practical test consisting of an objective, structured clinical examination is taken, in which standard patients are used and the professor directly assesses the level of skills acquired with a "real" case.

Key words: Problem-based learning. Teaching. Education. Undergraduate. Urology. 


\section{$\mathrm{L}$} as Facultades de Medicina se enfrentan actualmente a los cambios que se han planteado en la sociedad actual. Las nuevas necesidades derivadas de los actuales cambios sociales, económicos, tecnológicos, científicos, epidemiológicos, competitividad, globalización, etc..., han hecho que múltiples organismos nacionales e internacionales hayan sugerido a los estamentos educativos la necesidad de desarrollar cambios en las estrategias educativas orientados al desarrollo de un profesional "eficaz" y con competencias adaptadas a las necesidades sociales y sanitarias actuales $^{1}$. Las Facultades de Medicina no deben, por tanto, permanecer indiferentes a las importantes reformas sanitarias que espera la sociedad, aceptando que deben "rendir cuentas" y teniendo la certeza de que sus actuaciones, docentes y de investigación, tienen impacto en el entorno social en el que se encuentran. Para que muchas facultades reconozcan sus obligaciones de "rendir cuentas" a la sociedad, la OMS elaboró un documento en el que se recoge esta necesidad y propuso un marco de trabajo diseñado para ayudar a las facultades a evaluar sus progresos en este objetivo en función de cuatro valores: relevancia, coste-efectividad, calidad y equidad en la atención sanitaria $^{2}$. En este sentido, el papel del futuro médico en la sociedad cambia, pasando a ser el denominado "médico 5 estrellas":

- Prestador de atención sanitaria, considerando al individuo como integrante de una familia y de una comunidad.

- Decisor que determina las tecnologías aplicar desde un punto de vista ético y eficiente.

- Comunicador: debiendo de transmitir y promover eficazmente cambios de vida saludables.

- Líder comunitario que pueda emprender medidas en nombre de la comunidad.

- Gestor de los usos y recursos de salud disponibles.

En definitiva, todas estas circunstancias ponen de manifiesto la necesidad de introducir estos cambios dentro de las estrategias educativas en nuestras facultades. Estas necesidades han sido también apoyadas por la Conferencia de Decanos (Granada 2001) en la que se recomienda a las facultades que incluyan en sus currículos la identificación de competencias finales en función de las necesidades sociales y cambios congruentes con el aprendizaje de adultos. Asimismo, en el Libro Blanco de las Profesiones Sanitarias de Cataluña se recoge la necesidad de que la educación pregrado sea más práctica, que responda a las necesidades sanitarias, interdisciplinaria, basada en la resolución de problemas y centrada en el estudiante ${ }^{3}$. Todos estos modelos llevan, sin duda alguna, a una integración vertical y horizontal de todas las materias a impartir, yendo en contraposición con el modelo clásico, persistente todavía en muchas de nuestras facultades, introducido por Flexner en 1910: primero ciencias básicas, posteriormente ciencias clínicas, enseñanza en hospitales (nada o poco en comunidad) y precediendo la teoría a la práctica.

El problema actualmente es definir cuales son las competencias que ese médico "cinco estrellas" debe adquirir durante su paso por la facultad. Existen muchos documentos al respecto, unos específicos de cada materia y otros más globales. Quizás de los mejores definidos están los especificados en el documento elaborado por los Decanos de las Facultades de Medicina escocesas ${ }^{4}$ en un intento de unificar a las facultades escocesas en identificar las competencias esenciales de aprendizaje. En este documento se establece qué es capaz de hacer el médico (hacer lo adecuado = inteligencia técnica) cómo el médico alcanza la práctica (hacer las cosas bien = inteligencia intelectual, emocional, analítica y creativa) y, finalmente el médico como profesional (la persona adecuada haciéndolo = inteligencia personal). El documento recoge una serie de competencias de lo que el médico debe ser capaz de hacer en cuanto a habilidades, competencias, manejo del paciente, comunicación, etc.

En este ambiente de cambios estructurales, nace la Facultad de Medicina de la Universidad de Castilla-La Mancha en el año 1998, habiendo terminado la primera promoción durante el curso 2003/2004. Su creación obedece a la necesidad de contar con una Facultad de Medicina en una Comunidad Autónoma compuesta de cinco provincias y con una población de mas de millón y medio de habitantes. Los propulsores de dicha facultad se comprometieron con los estamentos educativos en la creación de una facultad "diferente" con una metodología docente más acorde 
con las necesidades sociales actuales y dentro del entorno en el que nos encontramos, optándose por estrategias centradas en el estudiante y, entre ellas, con el aprendizaje basado en problemas ${ }^{5}$.

\section{ESTRATEGIAS EDUCATIVAS}

La selección de estrategias educativas en ciencias de la salud constituye una de las decisiones más importantes. Las estrategias más modernas se han considerado como polos opuestos a las clásicas o tradicionales, aunque, en realidad, una buena estrategia educativa no tiene porqué ser excluyente de otra existiendo múltiples gamas intermedias entre las que se puede elegir.

A la hora de formar un profesional de la medicina que sea capaz de trabajar en el medio comunitario, tendremos que ofertarle unas posibilidades de aprendizaje en dicho medio primando, por tanto, la enseñanza basada en la comunidad sobre la enseñanza basada en medios hospitalarios. Si por otra parte lo que se pretende es que solucione problemas deberemos proporcionarle igualmente una enseñanza basada en problemas. Estos conceptos sin embargo no deben considerarse como paradigmas y finalmente habrá que adaptar la enseñanza lo más posible a las competencias profesionales definidas previamente, diseñando las estrategias pedagógicas más adecuadas para alcanzar estos objetivos.

1. Enseñanza centrada en el estudiante o profesor:

En la primera, el estudiante es responsable de su propio aprendizaje y del camino para llegar a conseguirlo: "el estudiante aprende" y el profesor pasa a ser un tutor que guía y asesora a los estudiantes a rentabilizar el tiempo y la forma de obtención de la información. En la segunda, el profesor decide, dirige y controla el proceso de aprendizaje: "el profesor enseña".

2. Enseñanza centrada en transmisión de información o en solución de problemas:

La primera es la forma más habitual en nuestras facultades. El profesor transmite de forma ordenada y oral sus conocimientos a los alumnos. La segunda forma se aproxima más a la realidad del ejercicio profesional ya que la solución de problemas es una forma de estimular al estudiante para que éste adquiera, organice y utilice en un futuro sus conocimientos de manera similar a la forma en que debe resolver las situaciones que se le planteen en su ejercicio profesional.

3. Enseñanza integrada y enseñanza basada en disciplinas:

La enseñanza integrada es un sistema que implica un tipo de docencia en la que se sacrifica las características de las clásicas asignaturas integrando de cada una de ellas todo lo necesario para la comprensión de un problema "como un todo". La integración puede ser:

- Horizontal: coordinación de diferentes asignaturas en un solo curso

- Vertical: coordinación de diferentes asignaturas de distintos cursos.

La diferencia entre enseñanza integrada y la denominada "enseñanza coordinada" radica en que en la primera, las disciplinas tradicionales pierden su identidad para convertirse en una disciplina nueva.

En la enseñanza basada en disciplinas el conocimiento se estructura en base a la coherencia y metodología científica de un área en concreto de la ciencia, manteniendo las clásicas asignaturas su identidad.

4. Enseñanza basada en la comunidad y enseñanza basada en hospitales:

La primera enfatiza en los grupos de población o individuos teniendo en consideración las necesidades de la comunidad. La segunda, se enfoca en los hospitales y en los problemas de salud que se encuentran en ellos.

5. Enseñanza basada en cursos optativos o en programas estándar:

En la enseñanza basada en cursos optativos, el estudiante confecciona su propio currículo mediante la selección de materias dentro de la gama que se le oferte. En la basada en programas estándar, el currículo está prefijado de antemano

6. Enseñanza basada en programas sistemáticos u oportunistas:

Un programa sistemático implica que el alumno debe alcanzar unos conocimientos, actitudes y habilidades prefijadas, debiéndose establecer los objetivos de forma clara y los medios para lograrlos. En un sistema oportunista, no hay objetivos definidos y la enseñanza se organiza en función de las preferencias del 
profesorado o de las "oportunidades" que se presentan.

Como se comentó anteriormente, estas estrategias planteadas pueden llegar a representar extremos opuestos entre estrategias "modernas" y "continuistas o tradicionales". Cada una de ellas tienen sus ventajas y sus inconvenientes y entre ambas existen una serie de posibilidades intermedias entre las que se pueden optar y aplicar en función de las necesidades, características de la comunidad y recursos docentes disponibles.

\section{DESARROLLO DEL APRENDIZAJE BASADO EN PROBLEMAS}

Los métodos de enseñanza tradicional en la medicina han resultado eficaces durante el siglo XX. Sin embargo, la necesidad de implantar esta nueva metodología docente en nuestras facultades de medicina se justificaría más que por una nueva tendencia o moda, por la nueva atmósfera social, tecnológica y económica que hace que los egresados de las facultades de medicina deban poseer una serie de conocimientos que les hagan competentes a la hora de enfrentarse a los diversos problemas. Por tanto, el perfil del profesional que la sociedad requiere debe incluir:

- Capacidad de autoaprendizaje para toda la vida.

- Capacidad de análisis crítico de los problemas.

- Capacidad de utilización de avances tecnológicos.

- Capacidad de adaptación a los cambios en el ejercicio profesional.

- Garantía de calidad y fiabilidad de su trabajo.

Todas estas cualidades hacen que el futuro profesional sea capaz de recopilar, analizar y utilizar la cantidad inmensa de información actualizada que se genera hoy día. Esto último justifica la necesidad de que los estudiantes deban "aprender" a estar preparados a lo largo de su vida profesional, circunstancia que parece no estar debidamente tratada en los programas tradicionales más centrados en acumular durante 6 años toda la información que el futuro médico necesitará a lo largo de su vida profesional.

Dentro de las estrategias "modernas" se encuentra la enseñanza basada en problemas o aprendizaje por problemas (PBL). Esta estrategia, parafraseando al Prof. L Branda ${ }^{6}$ no es tan moderna y hay algunos ejemplos históricos que así lo reflejan. La enseñanza basada en PBL se fundamenta en el contexto del constructivismo ${ }^{7}$, mediante el cual el estudiante busca y selecciona la información, razona e integra los conocimientos previos y adquiridos, dando finalmente unas posibilidades diagnósticas y terapéuticas al problema planteado, tal y como se va a enfrentar en su actividad profesional. Los conocimientos previos y la actividad constituyen los pilares fundamentales del aprendizaje. Es decir, el "autodescubrimiento" es fundamental en esta estrategia educativa requiriendo una participación muy activa por parte del alumno: "el conocimiento no se transfiere de forma directa sino que el aprendizaje requiere la participación activa del alumno"8.

El PBL incorpora algunos de los aspectos de la psicología cognitiva como los desarrollados por Anderson $^{9}$, modelo cuya parte principal determina la naturaleza de las estructuras del conocimiento que se encuentran en la memoria activa, en los procesos de almacenamiento y recuperación de la información y los diversos factores que lo activan. Los resultados de estos trabajos dentro del campo de la psicología cognitiva se incorporan a la metodología del PBL con relación $\mathrm{a}^{1}$ :

- Adquisición de conocimientos: los estudiantes necesitan relacionar de forma activa los nuevos conocimientos con los que ya poseen.

- Transferencia de los conocimientos para resolver el problema. Dos condiciones favorecen esta transferencia

- El problema debe ser afrontado sin grandes conocimientos previos sobre el tema.

- Los estudiantes deben recibir un feedback inmediatamente durante la resolución del problema y no después.

- Autoaprendizaje continuado.

El PBL ha pasado de ser un método excepcional a ser la metodología docente empleada en más de 120 Facultades de Medicina en todo el mundo, tendencia que cada día es creciente. La metodología por PBL se desarrolló en primer lugar en los años sesenta en la Universidad de McMaster (Canadá). A nivel europeo, tras la implantación del método en 1974 en la Universidad de Maastrich (Holanda) son ya cerca de 20 facultades las que han implantado esta metodología docente, la cual también se imparte en otras disciplinas aparte de las ciencias de la salud. 


\section{APRENDIZAJE POR PROBLEMAS. CONCEPTO Y METODOLOGÍA}

El PBL es una metodología docente que integra varios de los conceptos definidos dentro de las estrategias de educación: aprendizaje centrado en el estudiante, integrado. basado en la solución de problemas y en la comunidad.

Este método utiliza casos de pacientes de los que el estudiante además de adquirir conocimientos en ciencias básicas y clínicas, debe desarrollar un razonamiento clínico para resolver el problema. La característica fundamental es que el problema debe ser planteado antes de que los estudiantes hayan aprendido los conceptos clínicos y básicos relacionados con el problema. Las características del método PBL incluyen ${ }^{10}$ :

- Trabajo en grupos pequeños.

- El papel del profesor se limita a ser un elemento facilitador en el proceso de análisis del problema y en el funcionamiento del grupo.

- Los estudiantes analizan el problema y seguidamente determinará las necesidades de aprendizaje.

- El estudio es individual.

- Finalmente: análisis y discusión en grupo y síntesis final.

Los objetivos que se pretenden conseguir con el método de PBL son:

- Aportar a los estudiantes competencias necesarias para el razonamiento clínico y resolución de problemas de manera que el estudiante perciba e interprete la información, genere hipótesis, adquiera cualidades de entrevista y competencia clínica para verificar o eliminar hipótesis, formule el problema a partir de las hipótesis resultantes y elabore el diagnóstico y tratamiento adecuado.

- Facilitar la adquisición y retención de conocimientos.

- Integrar las distintas disciplinas básicas y clínicas.

- Adquirir el hábito de autoaprendizaje.

- Incentivar el interés por aprender.

Un buen problema debe presentar el tipo de información que normalmente puede obtener el médico del paciente, debe permitir obtener al estudiante información de tipo secuencial, proporcionar múltiples enfoques con resultados diferentes en función de las estrategias desarro- lladas. Los problemas deben estar acompañados de material audiovisual. El planteamiento y resolución del problema se realiza en tres fases: - Fase 1 (5 etapas) (1,30 horas de duración).

Se realiza en grupo. Tras la lectura del problema el grupo, de forma organizada, clarifica los términos y datos del enunciado, define el problema y prepara una lista de elementos y fenómenos a explicar, proponiendo tantas explicaciones e hipótesis como sean necesarias (tormenta de ideas) y utilizando experiencias previas para razonar y proponer explicaciones e hipótesis. El grupo debe organizar y discutir las propuestas y finalmente formular los objetivos del aprendizaje. En esta fase el "profesor tutor" controlará que todos los términos importantes del problema sean comprendidos adecuadamente, que se concrete el contenido del problema diferenciado lo esencial de lo superfluo, que se logre un consenso sobre la lista y el orden de las cuestiones a discutir, controlar el tiempo y las discusiones primando el profundizar el nivel de discusión, mantener el grado de atención adecuado y controlar y "asesorar" a la hora de definir los objetivos de aprendizaje.

- Fase 2 (15 a 24 horas):

Autoaprendizaje. Es una fase de trabajo individual. Mediante estudio el estudiante completa los objetivos del aprendizaje planteados.

- Fase 3 (1,30 horas de duración).

Básicamente, durante esta fase se analizan y discuten los conocimientos adquiridos aplicados al problema y el "profesor tutor" verificará que los objetivos de aprendizaje se hayan cumplido e intentará mantener el nivel de atención del grupo.

\section{APRENDIZAJE DE LA UROLOGÍA BASADO EN PROBLEMAS EN LA FACULTAD DE MEDICINA DE LA UNIVERSIDAD DE CASTILLA-LA MANCHA}

La urología forma parte de una asignatura troncal denominada Patología Médica y Quirúrgica II que se imparte durante el quinto curso. El resto de materias que se incluyen en esta asignatura son: aparato digestivo, cirugía digestiva y endocrina, oncología médica y quirúrgica, endocrinología, nutrición y nefrología. 
La urología se desarrolla de forma integrada con nefrología. El número de alumnos por curso es aproximadamente 75 , los cuales está divididos en 5 grupos de 15-18 alumnos. La "rotación" de los alumnos tiene seis semanas de duración durante las cuales los estudiantes tienen una media de 2 horas diarias de teoría (ya sean de nefrología y/o urología) y el resto son rotatorias por las distintas actividades desarrolladas en los servicios: tres semanas en nefrología y tres semanas en urología. Los estudiantes reciben al iniciar su rotación un cuaderno donde vienen definidos los objetivos a cubrir, los problemas a resolver y los listados de talleres, seminarios y conferencias ${ }^{11}$.

Al finalizar la rotación los estudiantes realizan un examen teórico conjunto con 100 preguntas de respuestas múltiple (50 de urología y 50 de nefrología) y un examen práctico de las habilidades adquiridas durante su rotación. A final de curso existe otra prueba práctica con la utilización de pacientes estandarizados, previamente adiestrados, en la que cada estudiante de forma individual se enfrenta a un problema clínico, debiendo el profesor evaluar de forma directa el nivel de competencias adquiridas ante un "caso real" Esta evaluación de competencias, que se denomina OSCE (examen clínico objetivo y estructurado) se implantó en nuestra facultad para todas las materias clínicas a partir de cuarto curso. Pruebas similares han sido referidas en la literatura en relación a la evaluación de la enseñanza de la uro$\operatorname{logia}^{12}$. La nota final de la asignatura se obtiene ponderadamente de la siguiente forma: $40 \%$ examen teórico, 30\% examen práctico y 30\% examen ante pacientes simulados. En la evaluación práctica, además de calificar la actitud del estudiante con el paciente (habilidades de comunicación) se valora el hecho de que el estudiante interrogue, explore y solicite pruebas diagnósticas adecuadas para el diagnóstico del paciente.

Los contenidos que se desarrollan dentro de la urología son:

1) Semiología urológica y exploración del enfermo urológico.

2) Uropatía obstructiva.

3) Anomalías congénitas del riñón, de la vía excretora superior, vésicoureterales. genitales, reflujo vésicoureteral.

4) Traumatismos urológicos.
5) Infecciones del tracto urinario. Infecciones urinarias especificas e inespecíficas.

6) Cistopatías inflamatorias crónicas. Síndrome uretral.

7) Litiasis urinaria.

8) Tumores del parénquima renal.

9) Tumores uroteliales.

10) Hiperplasia benigna de la próstata. Cáncer de próstata

11) Tumores de pene y tumores escrotales. Lesiones premalignas.

12) Tumores testiculares.

13) Patología renal vascular y trasplante renal.

14) Vejiga neurógena. Disfunción vésicoesfinteriana. Incontinencia urinaria. Patología del suelo pélvico.

15) Varicocele. Hidrocele. Escroto agudo. Fimosis. Parafimosis. Priapismo.

16) Infertilidad masculina. Disfunción eréctil.

17) Patología quirúrgica suprarrenal. Tumores retroperitoneales.

Los objetivos generales que deben adquirir los estudiantes son desde un punto de vista teórico:

- Adquirir los conocimientos básicos sobre la epidemiologia, etiología, prevención e importancia socioeconómica de las distintas enfermedades que afectan al aparto urinario, en ambos sexos y al genital masculino, desarrolladas en los programas teóricos y prácticos.

- Conocer los trastornos fisiopatológicos, y clínicos de estas afecciones.

- Conocer los principios terapéuticos fundamentales de que dispone la urología y saber cuales son los más adecuados en cada caso.

- Reconocer la patología urológica que requiere atención urgente ya sea por el médico general o por el especialista.

Los objetivos a desarrollar desde un punto de vista práctico:

- Mostrar aptitud suficiente para practicar una correcta anamnesis y exploración clínica y física del enfermo urológico.

- Mostrar aptitud y habilidad suficiente para realizar maniobras urológicas instrumentales básicas: sondaje uretral, examen rectal, etc...

- Adquirir conocimientos para la lectura e interpretación de exploraciones radiológicas y otras pruebas complementarias básicas urológicas. 
Para adquirir estos conocimientos, la enseñanza de la urología en nuestra facultad se ha planteado en base al desarrollo de:

A) Enseñanza basada en problemas: Mediante el planteamiento y resolución de estos 7 problemas se pretenden adquirir los objetivos teóricos y prácticos acerca de los contenidos 5, 7, $8,9,10,12$ y 14.

- Dr. orino sangre.

- Dr. tengo fiebre, me escuece al orinar y me duelen los riñones.

- Dr. se me escapa la orina.

- Dr. he perdido peso, orino sangre y me duele el riñón.

- Dr. me toco un bulto en el testículo.

- Dr. tengo cólico al riñón.

- Dr. no puedo orinar.

B) Conferencias. Siguen la sistemática clásica de los métodos tradicionales. Se imparten un total de tres con una duración aproximada cada una de hora y media

1. Fisiopatología de la obstrucción del tracto urinario superior e inferior. Fibrosis retroperitoneal

2. Mecanismos reguladores de la micción, erección y eyaculación. Fisiología de la reproducción masculina.

3. Patología vascular renal. Hipertensión vasculorrenal.

C) Seminarios: Mediante este procedimiento se plantea corroborar y poner al día conocimientos previos que debe aportar el estudiante y que de forma individual o en grupo han adquirido a partir de las fuentes bibliográficas y de otro tipo que el profesor ha facilitado. Los seminarios que se imparten y los objetivos de aprendizaje que se intentan lograr hacen referencia a los objetivos de aprendizaje no cubiertos con problemas o conferencias.

D) Talleres de habilidades: Representan clases eminentemente prácticas destinadas a la adquisición de habilidades tanto manuales como de interpretación de pruebas diagnósticas básicas. Para ello se utilizan además de diferentes imágenes del archivo urológico, maniquíes de exploración urológica y sondaje vesical. Los talleres que se han planteado son:
1. Exploración física del aparato urinario y genital.

2. Exploraciones instrumentales en urología. Urodinámica. Sondaje vesical.

3. Diagnóstico radiológico en urología: Radio grafía simple y estudios con medios de contrastes (I y II).

4. Diagnóstico ecográfico, T.A.C. y R.N.M. en urología. Estudios con isótopos en urología.

5. Taller conjunto Nefro-Urología de interpretación de los datos de sedimento urinario y microbiológicos en nefrourología. Sistemática para la realización de un cultivo de orina fraccionado. Interpretación de un espermiograma y de una bioquímica seminal normal. Patrón hormonal normal del varón fértil.

Para desarrollar todas estas actividades se dispone de dos Hospitales Universitarios, tres profesores asociados clínicos y 10 profesores-tutores colaboradores.

\section{PROBLEMAS DEL APRENDIZAJE MEDIANTE PBL}

La metodología PBL tiene también sus detractores, habiéndose señalado varios inconvenientes:

- Uno de los aspectos que se ha argumentado es el hecho de que las facultades con metodología PBL no impartan todos los conocimientos que se desarrollan con los modelos tradicionales. Se ha comprobado que no existe vínculo directo entre lo que se enseña y lo que se aprende. De hecho, la media de conceptos que se suelen emitir por hora en una enseñanza tradicional es de 24 (siendo recomendado como mucho hasta 6) de los que los estudiantes tan solo retienen entre un 35-60\% y la mayor parte los habrán olvidado unos meses después del curso ${ }^{13}$.

- Otro aspecto en contra radica en que requiere de un profesorado experto y bien entrenado, cualidad que en una facultad joven, como la nuestra, está todavía por desarrollar. En este sentido, existen múltiples estrategias orientadas a facilitar el aprendizaje y entrenamiento en esta metodología pedagógica "activa" ${ }^{14}$.

- La enseñanza basada en PBL es mucho más compleja de planificar, de estructurar y necesita un gran número de recursos humanos y materiales para desarrollarla. Sin embargo, no parece 
existir una conclusión científica que sustente las ventajas de la enseñanza basada en problemas ${ }^{15}$, por lo que esta gran utilización de recursos no compensaría la escasa ventaja que la metodología PBL pudiera tener en la adquisición de conocimientos y en la práctica clínica en comparación con métodos convencionales. Estos autores sî reconocen que esta metodología proporciona mayor motivación del alumno por aprender.

- Los estudiantes aprenden a integrar ciencias básicas con clínicas pero este hecho parece que les incapacita para separar ambos campos cuando la resolución del problema lo requiere.

- Finalmente, Un problema no bien estructurado, conduce a los estudiantes a una situación "desestructurada", perdiéndose dentro de un mundo de posibilidades.

En definitiva, la metodología PBL es mucho más compleja de planificar, estructurar. Necesita un gran número de recursos humanos $\mathrm{y}$ materiales para desarrollarla que, como en el caso de nuestra facultad, no está plenamente contemplado fundamentalmente a nivel de enseñanza clínica requiriendo un inmenso sobreesfuerzo de profesorado (que deben coordinar su labor asistencial y docente) y en un gran número de colaboradores desinteresados que a la larga, lógicamente y pasadas las etapas de ilusión inicial, entraran en fases de "saturación y desánimo" a lo cual puede también contribuir los últimos cambios en la legislación universitaria en cuanto al acceso a plazas vinculadas.

\section{Agradecimientos}

A la Unidad de Educación Médica (UEM), en especial a su directora Dra. María Teresa Alfonso Roca y a su subdirectora Dña. Pilar Serrano Casarrubio. La labor de la UEM es indispensable para que todo este engranaje funcione correctamente. Sólo ellas saben de nuestros desvelos y esfuerzos para el desarrollo de este programa. Su empuje, entusiasmo, apoyo y asesoramiento en todo momento nos anima a seguir en ello.

\section{REFERENCIAS}

1. Alfonso Roca MT, Fonseca M. "Aprendizaje basado en problemas: situación actual y perspectivas de implantación en las facultades de medicina europeas". Dolor 2001 16:119127.
2. Boelen Ch, Heck JE. En: Definiendo y midiendo la capacidad de las facultades de medicina de rendir cuentas ante la sociedad. División de Desarrollo de Recursos Humanos y Fortalecimiento de la Capacidad. Organización Mundial de la Salud. Ginebra. 1997.

3. Pomes X, Oriol A, de Oleza R, Ania O, Avila A, Branda L, Brugulat P, Gual A, Creus M, Zurro AM. Le livre blanc des professions de sante de Catalogne. Cah Sociol Demogr Med 2003;43(3):341-355.

4. Grupo Médico Curricular de Decanos Escoceses Marzo 2000. Aprendizaje en competencias en el pregrado de Medicina en Escocia: Fundación para los médicos competentes y reflexivos. Traducido por el Grupo de Trabajo del Proyecto FIS de Evaluación de Tecnologias sanitarias 02/10031. Departamento de Neurociencias. Facultad de Medicina y Odontología. Universidad del País Vasco.

5. Memoria para la creación de la Facultad de Medicina de la Universidad de Castilla-La Mancha. Albacete, enero de 1998. Universidad de Castilla-La Mancha.

6. Branda L. "Aprendizaje basado en problemas, centrado en el estudiante, orientado a la comunidad. Universidad". Máster Internacional de Pedagogía Médica. Unidad de Educaciín Médica. Facultad de Medicina de la Universidad de Castilla-La Mancha. marzo 2003.

7. Giordan A, de Vecci G. "Les origines du savoir". Defachaux et Nieste. 1994.

8. Schmidt H. "Foundation of problem-based learning: some explanatory notes". Med Educ. 1993; 27:422-423.

9. Gardener H. "The mind's new science. A history of the cognitive evolution”. New York basic Books. 1985.

10. Alfonso Roca MT, Fonseca Alfonso M. "Aprendizaje en el medio clínico. Guía del profesor-tutor en el Aprendizaje por Problemas (APP)”. Facultad de Medicina. Universidad de Castilla-La Mancha

11. Unidad de Educación Médica. Patología Médica y Quirúrgica II. Guia del Alumno. Facultad de Medicina. Universidad de Castilla-La Mancha. http/www.medab.uclm.es

12. Sibert L, Grand'Maison P, Charlin B, Grise P. Evaluation de la competence clinique en urologie: approche innovatrice basee sur l'observation de la performance. Prog Urol 1997;7(4):581-589.

13. Anderson JJ, Graham A. A problem in medical education: is there an information overload?. Med Educ. 1980;14:4-7.

14. Chastonay P, Guilbert JJ, Rougemont A. The construction of a 'topic tree': a way of familiarizing a teaching staff to problem-oriented learning in a master's programme in public health. Med Educ 1991;25(5):405-413.

15. Colliver JA. Effectiveness of problem-basedl learning curricula: research and theory. Acad Med 2000;75(3):259-266.

Dr. A.S. Salinas Sánchez

Pablo Medina, $22-2^{\circ}$ A

02005 Albacete

e-mail: asalinas@pulso.com

(Trabajo recibido el 27 julio de 2004) 